\title{
The physics of crystallizing white dwarfs
}

\author{
J. Isern ${ }^{1}$, R. Mochkovitch² , E. García-Berro ${ }^{3}$, \\ and
}

M. Hernanz ${ }^{1}$

Received —

\footnotetext{
${ }^{1}$ Institute for Space Studies of Catalonia (CSIC Research Unit), Edifici Nexus-104, Gran Capità 2-4, 08034 Barcelona, Spain

${ }^{2}$ Institut d'Astrophysique de Paris, CNRS, 98bis Bd Arago, 75014 Paris, France

${ }^{3}$ Departament de Física Aplicada, Universitat Politècnica de Catalunya, Jordi Girona Salgado s/n, Mòdul B-5, Campus Nord, 08034 Barcelona, Spain
} 


\begin{abstract}
White dwarfs can be used as galactic chronometers and, therefore, provide important information about galactic evolution if good theoretical models of their cooling are available. Consequently, it is natural to wonder if all the sources or sinks of energy are correctly taken into account. One of these sources is partial differentiation of the chemical components of the white dwarf upon crystallization. In this paper we use a new formalism to show that if there is a redistribution of the elements inside the star, there is a net release of energy that has to be radiated away and that slows down the cooling rate of the white dwarf.
\end{abstract}

Subject headings: stars: interiors — white dwarfs. 


\section{Introduction}

The evolution of white dwarfs is essentially a cooling process that lasts for $\sim 10$ Gyr. Since the study of white dwarfs allows to obtain information about the age of the Galaxy (Winget et al. 1987, García-Berro et al. 1988a,b, Hernanz et al. 1994), it is important to identify all the sources of energy as well as the mechanisms that control its outflow.

The vast majority of white dwarfs (those with masses in the range $0.45 \lesssim M / M_{\odot} \lesssim 1.1$ ) are made of a mixture of carbon, oxygen and some impurities coming from the metal content of the parent star. The most important of these impurities is ${ }^{22} \mathrm{Ne}$, which results from He-burning on the ashes of the CNO cycle, and reaches $\sim 2 \%$ by mass in Population I stars. Since during the cooling process the star experiences a phase transition, it is natural to wonder if a change of solubility at the onset of crystallization can provide an extra source of energy (Schatzman 1958, Stevenson 1980, Mochkovitch 1983).

Segretain and Chabrier (1993) and Segretain et al. (1994) computed phase diagrams for arbitrary binary mixtures in terms of the modern density-functional theory of freezing. They showed that the shape of the phase diagram was completely characterized by the charge ratio of the mixture, $Z_{1} / Z_{2}$. Their diagrams evolve from the spindle form for $0.72 \leq Z_{1} / Z_{2}<1$, into an azeotropic form for $0.58 \leq Z_{1} / Z_{2}<0.72$ and finally into an eutectic form for $Z_{1} / Z_{2}<0.58$. Using these phase diagrams, Hernanz et al. (1994) showed that the chemical differentiation of pure carbon-oxygen mixtures could introduce a delay of up to $\sim 2$ Gyr, depending on the adopted initial profiles of carbon and oxygen in the white dwarf before freezing. The settling of ${ }^{22} \mathrm{Ne}$ was much more spectacular since it could keep the white dwarf warm during several billion years, if the abundance of neon was assumed to be $X_{22}=0.02$ (Isern et al. 1991, Segretain et al. 1994). The deposition of ${ }^{56} \mathrm{Fe}$, the second impurity in importance, turned out to be less important (Xu and Van Horn 1992, Segretain et al. 1994) because of its smaller abundance, $X_{56}=0.001$. In any case, and contrary to 
the carbon-oxygen case, these impurities should not affect the determination of the disk age from white dwarfs since their abundances are expected to be negligible in the oldest objects (Hernanz et al. 1994).

Although the importance of the mechanical and thermodynamical consequences of the solidification of alloys has been recognized in Geophysics (Loper 1978, Chen 1995), this is not the case in Astrophysics and very often these phenomena are either completely ignored or sometimes, misinterpreted. In this paper we reexamine the efficiency of convective mixing, which is responsible for the redistribution of the chemical elements during crystallization, and we provide a suitable formalism to compute the subsequent energy released during this process.

\section{The physics of the crystallization process}

Due to the spindle shape of the phase diagram of $\mathrm{C} / \mathrm{O}$ mixtures, the solid formed upon crystallization is richer in oxygen than the liquid and therefore denser. Using the condition of pressure continuity, the density excess can be estimated to be:

$$
\frac{\delta \rho}{\rho} \simeq-\frac{\delta P_{\mathrm{i}}}{\gamma P_{\mathrm{e}}}-\frac{\delta Y_{\mathrm{e}}}{Y_{\mathrm{e}}}
$$

where $P_{\mathrm{i}}$ and $P_{\mathrm{e}}$ are the ionic and electronic pressures respectively, $\gamma$ is the electron

adiabatic index and $Y_{\mathrm{e}}$ is the number of electrons per nucleon. For a $0.6 M_{\odot}$ white dwarf with equal amounts of carbon and oxygen, $\delta \rho / \rho \simeq 10^{-4}$. Therefore, as the solid core grows from the center of the star the lighter liquid left behind can be redistributed by Rayleigh-Taylor instabilities (Stevenson, 1980; Mochkovitch 1983).

The local energy budget of the white dwarf can be written as:

$$
\frac{d L_{\mathrm{r}}}{d m}=-\epsilon_{\nu}-T \frac{d S}{d t}
$$


where all the symbols have their usual meaning. Assume now that the white dwarf is made of two chemical species with atomic numbers $Z_{0}$ and $Z_{1}$, mass numbers $A_{0}$ and $A_{1}$, and abundances by mass $X_{0}$ and $X_{1}$, respectively $\left(X_{0}+X_{1}=1\right)$. In the following, the suffix 0 will always refer to the heavier component. Using the First Principle of Thermodynamics, the right hand side of equation (2) can be written as:

$$
\frac{d L_{\mathrm{r}}}{d m}=-\epsilon_{\nu}-P \frac{d V}{d t}-\frac{d E}{d t}
$$

where $E$ is the internal energy per unit mass and $V=1 / \rho$. We can also write

$$
\frac{d E}{d t}=\left(\frac{\partial E}{\partial T}\right)_{V, X_{0}} \frac{d T}{d t}+\left(\frac{\partial E}{\partial V}\right)_{T, X_{0}} \frac{d V}{d t}+\left(\frac{\partial E}{\partial X_{0}}\right)_{T, V} \frac{d X_{0}}{d t}
$$

Using the elementary thermodynamic relation

$$
\left(\frac{\partial E}{\partial V}\right)_{T, X_{0}}=-P+T\left(\frac{\partial P}{\partial T}\right)_{V, X_{0}}
$$

equation (2) can now be written as:

$$
-\left(\frac{d L_{\mathrm{r}}}{d m}+\epsilon_{\nu}\right)=C_{\mathrm{v}} \frac{d T}{d t}+T\left(\frac{\partial P}{\partial T}\right)_{V, X_{0}} \frac{d V}{d t}-l_{\mathrm{s}} \frac{d M_{\mathrm{s}}}{d t} \delta\left(m-M_{\mathrm{s}}\right)+\left(\frac{\partial E}{\partial X_{0}}\right)_{T, V} \frac{X_{0}}{d t}
$$

where $l_{\mathrm{s}}$ is the latent heat of crystallization and $\dot{M}_{\mathrm{s}}$ is the rate at which the solid core grows; the delta function indicates that the latent heat is released at the solidification front. Notice that chemical differentiation contributes to the luminosity not only through compressional work, which is negligible, but also through the change in the chemical abundances, which leads to the last term of this equation. Notice, as well, that the largest contribution to $L_{\mathrm{r}}$ due to the change in $E$ exactly cancels out the $P d V$ work for any evolutionary change (with or without a compositional change). This is, of course, a well known result (Mestel 1952, Kovetz and Shaviv 1975, Lamb and Van Horn 1975, D'Antona and Mazzitelli 1990) that can be related to the release of gravitational energy (see below). 
Finally, integrating (6) over the whole star, we obtain:

$$
\begin{aligned}
L+L_{\nu} & =-\int_{0}^{M_{\mathrm{WD}}} C_{\mathrm{v}} \frac{d T}{d t} d m-\int_{0}^{M_{\mathrm{WD}}} T\left(\frac{\partial P}{\partial T}\right)_{V, X_{0}} \frac{d V}{d t} d m \\
& +l_{\mathrm{s}} \frac{d M_{\mathrm{s}}}{d t}-\int_{0}^{M_{\mathrm{WD}}}\left(\frac{\partial E}{\partial X_{0}}\right)_{T, V} \frac{d X_{0}}{d t} d m
\end{aligned}
$$

The first term of equation (7) is the well known contribution of the heat capacity of the star to the total luminosity (Mestel 1952). The second term represents the contribution to the luminosity due to the change of volume. It is in general small since only the thermal part of the electronic pressure, the ideal part of the ions and the Coulomb terms other than the Madelung term contribute (Kovetz and Shaviv 1975, Lamb and Van Horn 1975). However, when the white dwarf enters into the Debye regime, this term provides about the $80 \%$ of the total luminosity preventing the sudden disappearence of the star (D'Antona and Mazzitelli 1990). The third term represents the contribution of the latent heat to the total luminosity at freezing. Since the latent heat of Coulomb plasmas is small $\left(\sim k T_{\mathrm{s}} /\right.$ nucleus, where $T_{\mathrm{s}}$ is the solidification temperature), its contribution to the total luminosity is modest although not negligible. The fourth term represents the energy released by the chemical readjustement of the white dwarf, i.e. the release of the energy stored in the form of chemical potentials. This term is usually negligible in normal stars, since it is much smaller than the energy released by nuclear reactions, but it must be taken into account when all other energy sources are small.

The last term in equation (7) can be further expanded. Consider that the crystallization front is at mass coordinate $M_{\mathrm{s}}$, that in a time interval $\delta t$ the crystallization front advances by $\delta M_{\mathrm{s}}$, and that the extension of the convective shell driven by the Rayleigh-Taylor instability is $\Delta M$. Then the change in the chemical abundance of the mixing region $\left(\delta X_{0}^{\text {liq }}\right)$ can be related (assuming perfect mixing, see $\S 3$ below) to the difference between the chemical abundances of the liquid and the solid:

$$
\delta M_{\mathrm{s}}\left(X_{0}^{\mathrm{sol}}-X_{0}^{\mathrm{liq}}\right)=-\Delta M \delta X_{0}^{\mathrm{liq}}
$$


Besides, we can write

$$
\int_{0}^{M_{\mathrm{WD}}}\left(\frac{\partial E}{\partial X_{0}}\right)_{T, V} \delta X_{0} d m=\left(\frac{\partial E}{\partial X_{0}}\right)_{M_{\mathrm{s}}}\left(X_{0}^{\mathrm{sol}}-X_{0}^{\mathrm{liq}}\right) \delta M_{\mathrm{s}}+\delta X_{0}^{\mathrm{liq}} \int_{\Delta M}\left(\frac{\partial E}{\partial X_{0}}\right)_{T, V} d m
$$

where $\left(\partial E / \partial X_{0}\right)_{M_{\mathrm{s}}}$ is the partial derivative evaluated at the edge of the solid core. Taking into account equation (8), we can now write

$$
\int_{0}^{M_{\mathrm{WD}}}\left(\frac{\partial E}{\partial X_{0}}\right)_{T, V} \frac{d X_{0}}{d t} d m=\left(X_{0}^{\mathrm{sol}}-X_{0}^{\mathrm{liq}}\right)\left[\left(\frac{\partial E}{\partial X_{0}}\right)_{M_{\mathrm{s}}}-\left\langle\frac{\partial E}{\partial X_{0}}\right\rangle\right] \frac{d M_{\mathrm{s}}}{d t}
$$

where

$$
\left\langle\frac{\partial E}{\partial X_{0}}\right\rangle=\frac{1}{\Delta M} \int_{\Delta M}\left(\frac{\partial E}{\partial X_{0}}\right)_{T, V} d m
$$

From equation (10) we can define the total energy released per gram of crystallized matter due to the change in chemical composition as:

$$
\epsilon_{\mathrm{g}}=-\left(X_{0}^{\mathrm{sol}}-X_{0}^{\mathrm{liq}}\right)\left[\left(\frac{\partial E}{\partial X_{0}}\right)_{M_{\mathrm{s}}}-\left\langle\frac{\partial E}{\partial X_{0}}\right\rangle\right]
$$

The square bracket is negative since $\left(\partial E / \partial X_{0}\right)$ is negative and essentially depends on the density, which monotonically decreases outwards. The internal energy per unit mass can be divided into the electronic and the ionic components, so we can write $\epsilon_{\mathrm{g}}=\epsilon_{\mathrm{e}}+\epsilon_{\mathrm{i}}$. For the sake of simplicity we are only going to use here the completely degenerate nonrelativistic expression for the electrons

$$
E_{\mathrm{e}}=\frac{3}{2} K_{1} \rho^{2 / 3} Y_{\mathrm{e}}^{5 / 3}
$$

where $K_{1}=1.004 \times 10^{13}$ (cgs units), and the ideal contribution plus the Madelung term of the Coulomb energy for the ions

$$
E_{\mathrm{i}} \simeq \frac{\Re T}{\mu}\left(\frac{3}{2}-0.9 \Gamma\right)
$$

where $\Re$ is the gas constant and $\Gamma=\Gamma_{\mathrm{e}} \overline{Z^{5 / 3}}$ is the Coulomb coupling constant, with $\Gamma_{\mathrm{e}}=2.272 \times 10^{5}\left(\rho Y_{\mathrm{e}}\right)^{1 / 3} / T$ 
Since the contribution to the electron mole number of carbon and oxygen are very similar $\epsilon_{\mathrm{g}}$ is dominated by the ionic contribution (see $\S 4$ below). Thus, we can obtain a more transparent expression for $\epsilon_{\mathrm{g}}$ showing that it also corresponds to a release of gravitational energy. We first write

$$
\epsilon_{\mathrm{g}}=-\alpha\left(X_{0}^{\mathrm{sol}}-X_{0}^{\mathrm{liq}}\right)\left(\frac{\partial E}{\partial X_{0}}\right)_{M_{\mathrm{s}}}
$$

where we have introduced the parameter

$$
\alpha=\frac{\left(\frac{\partial E}{\partial X_{0}}\right)_{M_{\mathrm{s}}}-\left\langle\frac{\partial E}{\partial X_{0}}\right\rangle}{\left(\frac{\partial E}{\partial X_{0}}\right)_{M_{\mathrm{s}}}} \lesssim 1
$$

The ionic pressure associated to the Madelung energy is $P_{\mathrm{i}}=\frac{1}{3} \rho E_{\mathrm{i}}$ so that

$$
\epsilon_{\mathrm{g}} \simeq-3 \alpha\left(X_{0}^{\mathrm{sol}}-X_{0}^{\mathrm{liq}}\right) \frac{1}{\rho}\left(\frac{\partial P_{\mathrm{i}}}{\partial X_{0}}\right)_{M_{\mathrm{s}}} \simeq-3 \alpha \frac{\delta P_{\mathrm{i}}}{\rho}=3 \alpha \gamma \frac{P_{\mathrm{e}}}{\rho} \frac{\delta \rho}{\rho}
$$

$\delta P_{\mathrm{i}}$ being the change of ionic pressure at crystallization. The last term in the above equation has been obtained by taking into account pressure continuity, which leads to $\delta P_{\mathrm{i}}=-\delta P_{\mathrm{e}} \simeq-\gamma P_{\mathrm{e}} \delta \rho / \rho$. Finally, using the virial theorem in the form

$$
3\left\langle\frac{P_{\mathrm{e}}}{\rho}\right\rangle \simeq-\frac{\Omega}{M_{\mathrm{WD}}} \simeq \beta \frac{G M_{\mathrm{WD}}}{R_{\mathrm{WD}}} \simeq \beta g_{\mathrm{WD}} R_{\mathrm{WD}}
$$

where the average is taken over the white dwarf mass and where $\Omega=-\beta G M_{\mathrm{WD}} / R_{\mathrm{WD}}^{2}, g_{\mathrm{WD}}$ and $R_{\mathrm{WD}}$ are, respectively, the white dwarf gravitational energy, surface gravity and radius $(\beta=6 / 7$ for $\gamma=5 / 3)$, we obtain

$$
\epsilon_{\mathrm{g}} \simeq \alpha \beta \gamma \frac{P_{\mathrm{e}} / \rho}{\left\langle P_{\mathrm{e}} / \rho\right\rangle} g_{\mathrm{WD}} R_{\mathrm{WD}} \frac{\Delta \rho}{\rho} \simeq \mathrm{k} g_{\mathrm{WD}} R_{\mathrm{WD}} \frac{\Delta \rho}{\rho}
$$

The factor $\mathrm{k}$ is of the order of unity except close to the surface of the white dwarf where $\rho$ rapidly decreases. With this expression for $\epsilon_{\mathrm{g}}$, it is clearly seen that it corresponds to a release of gravitational energy. 


\section{The efficiency of the mixing process}

Before computing the energy released by chemical segregation it is convenient to check the efficiency of convective mixing. Let $v_{\text {crys }}$ be the propagation velocity of the solidification front into the $\mathrm{C} / \mathrm{O}$ mixture. This velocity, which can be obtained from the models, is very small, $\sim 0.1 \mathrm{~cm} / \mathrm{yr}$. The mass flux of carbon released by the front in the liquid phase is:

$$
F_{\mathrm{C}}^{\mathrm{crys}}=\rho v_{\text {crys }}\left(X_{\mathrm{C}}^{\mathrm{l}}-X_{\mathrm{C}}^{\mathrm{s}}\right)=\rho v_{\text {crys }} \Delta X_{\mathrm{C}}
$$

where $X_{\mathrm{C}}^{\mathrm{l}}$ and $X_{\mathrm{C}}^{\mathrm{s}}$ are the carbon mass fractions in the liquid and the solid respectively. The criterion for convective instability taking into account heat conduction from the convective eddies is (Stevenson and Salpeter 1977):

$$
\chi>k \epsilon
$$

with

$$
\begin{gathered}
\chi=-\frac{H_{\mathrm{P}}}{\rho c_{\mathrm{s}}^{2}} \mu\left(\frac{\partial P}{\partial \mu}\right)_{\rho, T}\left(\frac{1}{\mu} \frac{d \mu}{d r}\right) \\
\epsilon=\frac{H_{\mathrm{P}}}{\rho c_{\mathrm{s}}^{2}} T\left(\frac{\partial P}{\partial T}\right)_{\rho, \mu}\left[\frac{1}{T} \frac{d T}{d r}-\left(\Gamma_{3}-1\right) \frac{1}{\rho} \frac{d \rho}{d r}\right] \\
k=\frac{\tau_{\text {cond }}}{\tau_{\text {cond }}+\tau_{\text {conv }}}
\end{gathered}
$$

where $H_{\mathrm{P}}$ is the pressure scale height, $c_{\mathrm{s}}$ is the sound velocity, $\mu$ is the mean molecular weight, $\left(\Gamma_{3}-1\right)=(\partial \log T / \partial \log \rho)_{\text {ad }}$, and $\tau_{\text {cond }}$ and $\tau_{\text {conv }}$ are the conductive and the

convective characteristic times, respectively. If $l$ is the mixing length, the convective velocity can be written as

$$
v_{\text {conv }}=c_{\mathrm{S}}(\chi-k \epsilon)^{1 / 2} \frac{l}{H_{\mathrm{P}}}
$$

and the characteristic times are given by:

$$
\tau_{\mathrm{conv}}=\frac{l}{v_{\mathrm{conv}}}
$$




$$
\tau_{\text {cond }}=\frac{l^{2}}{K_{T}}
$$

where $K_{T}$ is the thermal conductivity. If the carbon mass flux released at the crystallization front is to be efficiently carried by convection we have

$$
F_{\mathrm{C}}^{\mathrm{cryst}}=F_{\mathrm{C}}^{\mathrm{conv}}=\rho v_{\mathrm{conv}}\left|\frac{d X_{\mathrm{C}}}{d r}\right| l
$$

together with a small superadiabaticity

$$
\chi-k \epsilon \sim 0
$$

If the superadiabaticity is indeed small (which will have to be checked on the final results), the gradient of carbon mass fraction in the white dwarf is given by

$$
\left|\frac{d X_{\mathrm{C}}}{d r}\right|=k\left|\frac{d X_{\mathrm{C}}}{d r}\right|_{\mathrm{ad}}
$$

where

$$
\left|\frac{d X_{\mathrm{C}}}{d r}\right|_{\mathrm{ad}}=-\frac{48}{\mu^{2}} \frac{\left(\Gamma_{3}-1\right) T\left(\frac{\partial P}{\partial T}\right)_{\rho, \mu}}{\left(\frac{\partial P}{\partial \mu}\right)_{\rho, T}}\left|\frac{1}{\rho} \frac{d \rho}{d r}\right|=\mathrm{Q}\left|\frac{1}{\rho} \frac{d \rho}{d r}\right|
$$

The value of $\mathrm{Q}$ has been computed for a $0.6 M_{\odot}$ crystallizing $\mathrm{C} / \mathrm{O}$ white dwarf and is typically a few $10^{-2}$. Now it is possible to obtain the Peclet number, $\mathrm{P}=\tau_{\text {cond }} / \tau_{\text {conv }}$, as

$$
\frac{\mathrm{P}^{2}}{\mathrm{P}+1} \simeq \frac{5}{3} \frac{v_{\text {crys }} \Delta X_{\mathrm{C}} H_{\mathrm{P}}}{K_{T} \mathrm{Q}} \simeq 0.1\left(\frac{v_{\text {crys }}}{0.1 \mathrm{~cm} / \mathrm{yr}}\right)\left(\frac{\Delta X_{\mathrm{C}}}{0.1}\right)\left(\frac{100 \mathrm{~cm}^{2} \mathrm{~s}^{-1}}{K_{T}}\right)\left(\frac{H_{\mathrm{P}}}{10^{9} \mathrm{~cm}}\right)
$$

where $K_{\mathrm{T}}=100 \mathrm{~cm}^{2} \mathrm{~s}^{-1}$ is a typical value of the conductivity in the white dwarf. Then $P \lesssim 1$ and the gradient of carbon mass fraction

$$
\left|\frac{d X_{\mathrm{C}}}{d r}\right| \simeq 4 \times 10^{-12}\left(\frac{v_{\text {crys }}}{0.1 \mathrm{~cm} / \mathrm{yr}}\right)\left(\frac{\Delta X_{\mathrm{C}}}{0.1}\right)\left(\frac{100 \mathrm{~cm}^{2} \mathrm{~s}^{-1}}{K_{T}}\right)\left(\frac{1}{\mathrm{P}}\right)
$$

is so small that $X_{\mathrm{C}}$ varies by less than $1 \%$ in the convective region.

We now check that the small superadiabaticity hypothesis is correct, i.e.:

$$
\chi-k \epsilon \ll \chi
$$


We first compute $\chi$ with the simplified equation of state described in $\S 2$. For a crystallizing $\mathrm{C} / \mathrm{O}$ white dwarf we get

$$
\chi \sim 10^{-4}\left(\frac{H_{\mathrm{P}}}{10^{9} \mathrm{~cm}}\right)\left(\frac{3 \times 10^{8} \mathrm{~cm} \mathrm{~s}^{-1}}{c_{\mathrm{s}}}\right)^{-2}\left(\frac{\left|\frac{d X_{\mathrm{C}}}{d r}\right|}{4 \times 10^{-12}}\right)
$$

An upper limit of the superadiabacity can be obtained by assuming that, due to the interaction of convection with rotation, the Rossby number

$$
R_{0}=\frac{v_{\mathrm{conv}}}{\omega l}
$$

is equal to unity, where $\omega$ is the angular velocity of the white dwarf (Stevenson and Salpeter 1976). Then,

$$
\chi-k \epsilon=\left(\frac{H_{\mathrm{P}} \omega}{c_{\mathrm{s}}}\right)^{2}=3 \times 10^{-7}\left(\frac{H_{\mathrm{P}}}{10^{9} \mathrm{~cm}}\right)^{2}\left(\frac{c_{\mathrm{s}}}{3 \times 10^{8} \mathrm{~cm} \mathrm{~s}^{-1}}\right)^{-2}\left(\frac{\Pi}{10 \mathrm{~h}}\right)
$$

where $\Pi$ is the rotation period. We therefore conclude that, even when rotation is

considered, convection is indeed an efficient mechanism to redistribute the carbon rich fluid out from the crystallization front and that the liquid phase can be considered well mixed.

\section{Consequences on white dwarf cooling}

Figure 1 displays the evolution of the energy released per unit mass crystallized as the solidification proceeds. For the sake of simplicity we have assumed in these calculations that the white dwarf is made of an homogeneous 50:50 (by mass) mixture of carbon and oxygen.

\section{EDITOR: PLACE FIGURE 1 HERE.}

The energy released near the center of the white dwarf is $\epsilon_{\mathrm{g}}=3.54 \times 10^{13} \mathrm{erg} / \mathrm{g}$ and the partial contribution of electrons and ions are, respectively $\epsilon_{\mathrm{e}}=-5.00 \times 10^{12} \mathrm{erg} / \mathrm{g}$ and 
$\epsilon_{\mathrm{i}}=4.04 \times 10^{13} \mathrm{erg} / \mathrm{g}$. The electron term is negative and different from zero only due to the mass defect of oxygen $\left(A_{0}=16-3.18 \times 10^{-4}\right)$ relative to carbon. If we had considered other species with a higher number of neutrons as compared with protons, as is the case of ${ }^{22} \mathrm{Ne}$ or ${ }^{56} \mathrm{Fe}$, the situation would have been the reverse. The total energy released during this process is $1.95 \times 10^{46} \mathrm{erg}$.

Since, to a good approximation, the luminosity of a white dwarf can be considered to be a function of the temperature of its nearly isothermal core, $T_{\mathrm{c}}$, it is possible to estimate the delay introduced by the solidification as:

$$
\Delta t=\int_{0}^{M_{\mathrm{WD}}} \frac{\epsilon_{\mathrm{g}}\left(T_{\mathrm{c}}\right)}{L\left(T_{\mathrm{c}}\right)} d m
$$

where $T_{\mathrm{c}}(m)$ is the core temperature when the crystallization front is located at $m$. The extra time to reach a luminosity $\log \left(L / L_{\odot}\right)=-4.5$ is $1.81 \mathrm{Gyr}$. To compute it we have used the same relationship between the luminosity and the core temperature as in Segretain et al. (1994). Of course, the total delay essentially depends on the transparency of the envelope. Any change in one sense or another can amplify or damp the influence of solidification in the cooling of white dwarfs and for the moment there are not completely reliable envelope models at low luminosities (Mazzitelli 1994).

\section{Conclusions}

We have provided a new formulation of the thermodynamics of phase separation upon crystallization that proves that chemical differentiation results in a net release of heat that is radiated away thus delaying the cooling of the star. This extra heating is not due to compressional work, but to the local changes of chemical abundances. This formulation is very simple and is suitable for introduction in evolutionary codes.

We have also shown that the hypothesis of perfect rehomogeneization of the liquid is 
reasonable and can be used to simplify the problem. Notice that if this was not the case, this would result in a decrease of the efficiency of the redistribution process as an energy source but would not invalidate the result that if there is some redistribution there is a release of heat.

Finally, we want to emphasize that the total delay introduced by this extra source of energy depends on the phase diagrams, on the initial chemical compositions and on the transparency of the outer envelope. Any change in these factors can produce noticeable changes in the final outcome.

Acknowledgements This work has been supported by the DGICYT grants PB94-0111, PB94-0827-C02-02, by the CIRIT grant GRQ94-8001 and by the AIHF237B. 


\section{REFERENCES}

Chen, C. F. 1995 J. Fluid Mech. 293, 81

D’Antona, F., Mazzitelli, I. 1990 ARA\&A, 28, 139

García-Berro, E., Hernanz, M., Mochkovitch, R., Isern, J. 1988a, A\&A, 193, 141

García-Berro, E., Hernanz, M., Isern, J., Mochkovitch, R. 1988b, Nature, 333, 642

Hernanz, M., García-Berro, E., Isern, J., Mochkovitch, R., Segretain, L., Chabrier, G. 1994, ApJ, 434, 632

Isern, J., Mochkovitch, R., García-Berro, E., Hernanz, M. 1991, A\&A, 241, L29

Kovetz, A., Shaviv, G. 1976, A\&A, 52, 403

Lamb, D.Q., Van Horn, H. M. 1975, ApJ, 200, 306

Liebert, J., Dahn C.C., Monet D.E. 1988, ApJ, 332, 891

Loper, D.E. 1978, Geophys. J. R. Astron. Soc. 54, 389

Mazzitelli, I. 1994, in IAU Col. 147: "The Equation of State in Astrophysics", Eds. G. Chabrier and E. Schatzman, p. 144

Mestel, L. 1952, MNRAS, 112, 583

Mochkovitch, R. 1983, A\&A, 122, 212

Schatzman, E. 1958, in "Hdb. d. Phys.", Vol. 51, S. Flüge editor, (Berlin: Springer Verlag)

Segretain, L., Chabrier, G. 1993, A\&A, 271, L13

Segretain, L., Chabrier, G., Hernanz, M., García-Berro, E., Isern, J., Mochkovitch, R. 1994, ApJ, 434, 641

Stevenson, D.J. 1980, J. Phys. Suppl. No 3, 41, C2-53

Stevenson, D.J., Salpeter, E.E. 1976, in "Jupiter", Ed. T. Gehrels, The University of Arizona Press, p. 85. 
Stevenson, D.J., Salpeter, E.E. 1977, ApJS, 35, 239

Winget, D. E., Hansen, H. J., Liebert, J., Van Horn, H. M., Fontaine, G., Nather, R.E., Kepler, S.O., Lamb, D.Q. 1987, ApJ, 307, 659

Xu , Van Horn, H.M. 1992, ApJ, 367, 662 
Fig. 1.- Energy released by the redistribution of elements per unit of crystallized mass (continuous line) and the corresponding time delay in the cooling (dotted line) as a function of the solid mass (lower panel) and the luminosity (upper panel). 

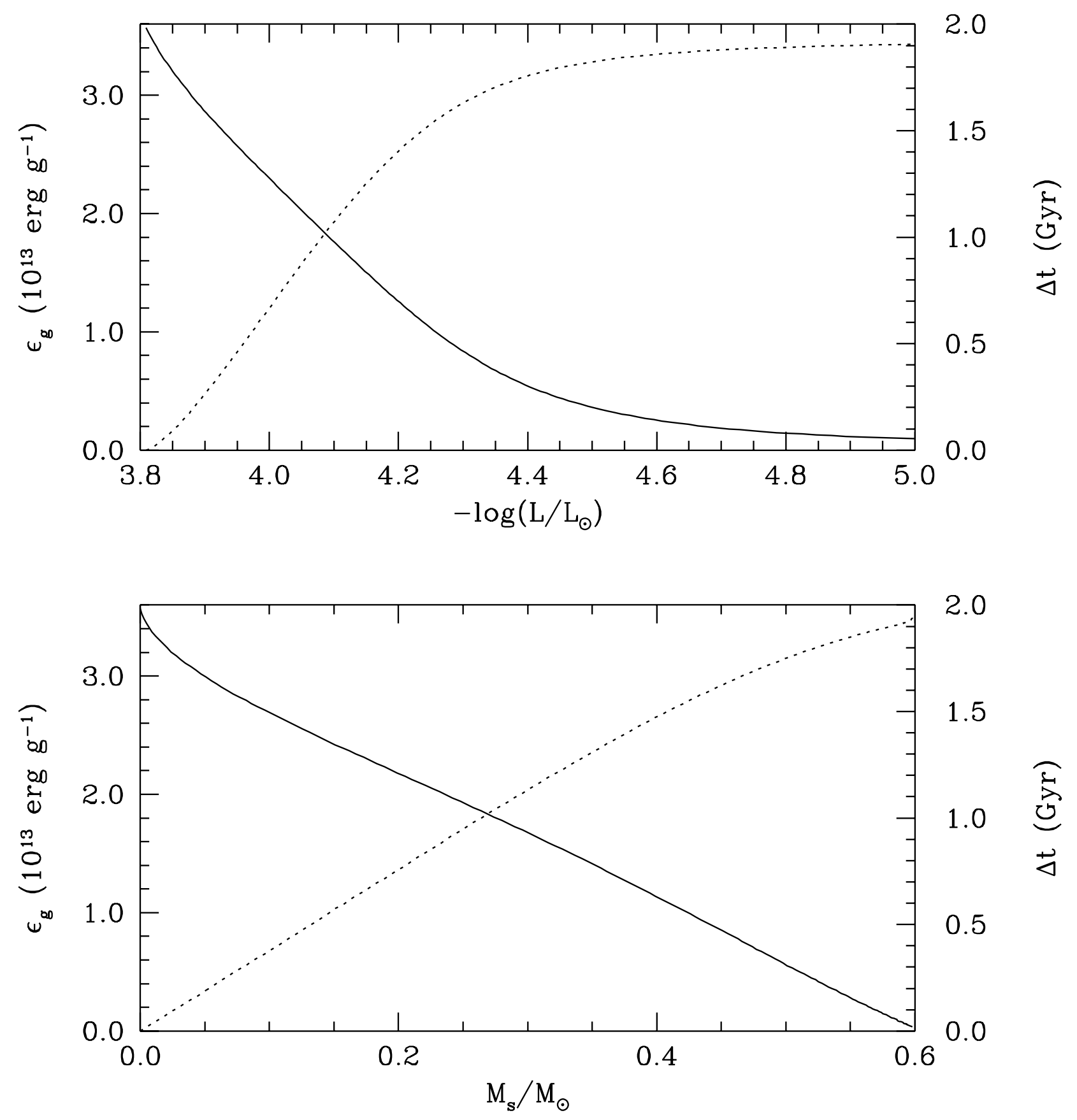